\title{
Ultralow Surface Resistance via Vacuum Heat Treatment of Superconducting Radio-Frequency Cavities
}

\author{
S. Posen $\odot,{ }^{*}$ A. Romanenko, A. Grassellino, O.S. Melnychuk®, and D.A. Sergatskov \\ Fermi National Accelerator Laboratory, Batavia, Illinois, 60510, USA
}

(Received 22 July 2019; revised manuscript received 6 November 2019; published 14 January 2020)

\begin{abstract}
We report on an effort to improve the performance of superconducting radiofrequency cavities by using heat treatment in a temperature range sufficient to dissociate the natural surface oxide. We find that the residual resistance is decreased significantly, and we find an unexpected reduction in the Bardeen-CooperSchrieffer (BCS) resistance. Together these result in extremely high-quality factor values at relatively large accelerating fields $E_{\text {acc }} \sim 20 \mathrm{MV} / \mathrm{m}: Q_{0}=3-4 \times 10^{11}$ at $<1.5 \mathrm{~K}$ and $Q_{0} \sim 5 \times 10^{10}$ at $2.0 \mathrm{~K}$. In one cavity, measurements of surface resistance versus temperature showed an extremely small residual resistance of just $0.63 \pm 0.06 \mathrm{n} \Omega$ at $16 \mathrm{MV} / \mathrm{m}$. Secondary ion mass spectrometry measurements confirm that the oxide is dissociated significantly, but they also show the presence of nitrogen after heat treatment. We also present studies of surface oxidation via exposure to air and to water, as well as the effects of very light surface removal via HF rinse. The study is performed on $1.3 \mathrm{GHz}$ cavities, but the effect may be extendable to other frequencies as well. The possibilities for applications and the planned future development are discussed.
\end{abstract}

DOI: 10.1103/PhysRevApplied.13.014024

\section{INTRODUCTION}

Superconducting radio-frequency (SRF) cavities are used widely in modern particle accelerator facilities (for examples of recent and near-future large SRF accelerators, see Refs. [1-6]) to transfer energy to beams of charged particles. By treating the niobium walls of the cavities with state-of-the-art surface processing techniques and cooling them to approximately $2 \mathrm{~K}$, it is possible to reach accelerating fields $E_{\text {acc }}$ in the scale of tens of megavolts per meter while maintaining a quality factor $Q_{0}$ in the $10^{10}$ range.

As progress in research and development pushes $Q_{0}$ and $E_{\text {acc }}$ higher, a given investment in an accelerator facility can result in beam parameters that are improved compared with what would have been possible with the previous state of the art. For example, processing cavities with the $120^{\circ} \mathrm{C}$ bake treatment $[7,8]$ enables the high beam energy of the European XFEL [1] and processing cavities with nitrogen doping [9] enables the high duty factor of LCLS-II [2] (see typical examples of these treatments in Fig. 1). To be realizable in applications, new treatments must not just achieve high $Q_{0}$ and $E_{\text {acc }}$ values, but must do so with high reproducibility. Nitrogen infusion is an example of a treatment that shows extremely promising $Q_{0}$ and $E_{\text {acc }}$ performance

\footnotetext{
*sposen@fnal.gov
}

Published by the American Physical Society under the terms of the Creative Commons Attribution 4.0 International license. Further distribution of this work must maintain attribution to the author(s) and the published article's title, journal citation, and DOI. in several tests [10], but has had limited reproducibility in some laboratories $[11,12]$. The process starts with a hightemperature heat treatment of a cavity in a vacuum furnace at approximately $800^{\circ} \mathrm{C}$, which is expected to dissolve the natural surface oxide layer. The furnace temperature is then lowered to $100-200{ }^{\circ} \mathrm{C}$, and low-pressure nitrogen gas is injected into the furnace. After several hours of the low-temperature nitrogen treatment on the post-oxide dissolution surface, secondary ion mass spectrometry (SIMS) measurements show that nitrogen interstitials can be found several tens of nanometers deep [10]. Unlike most other state-of-the-art cavity preparation methods, no material removal (e.g., via electropolishing (EP)) is done after furnace treatment. This likely makes it extremely sensitive to the pumping system and cleanliness of the furnace in which the infusion is performed.

To try to improve reliability, we developed a method to perform a nitrogen infusionlike treatment of a cavity after it has been assembled to vacuum hardware in a cleanroom environment. During the heat treatment, the inner cavity surface is expected to be exposed only to carefully cleaned and assembled surfaces, rather than to a vacuum furnace. The method has the added benefit of making it possible to measure cavity performance after the oxide dissolution step, but before the surface oxide regrows. Several initial tests are performed on $1.3 \mathrm{GHz}$ single-cell cavities after only the oxide dissolution step, and before adding a low-temperature step with nitrogen. The results of these initial tests, as well as a test with the addition of the low-temperature nitrogen step, are presented in this paper. 


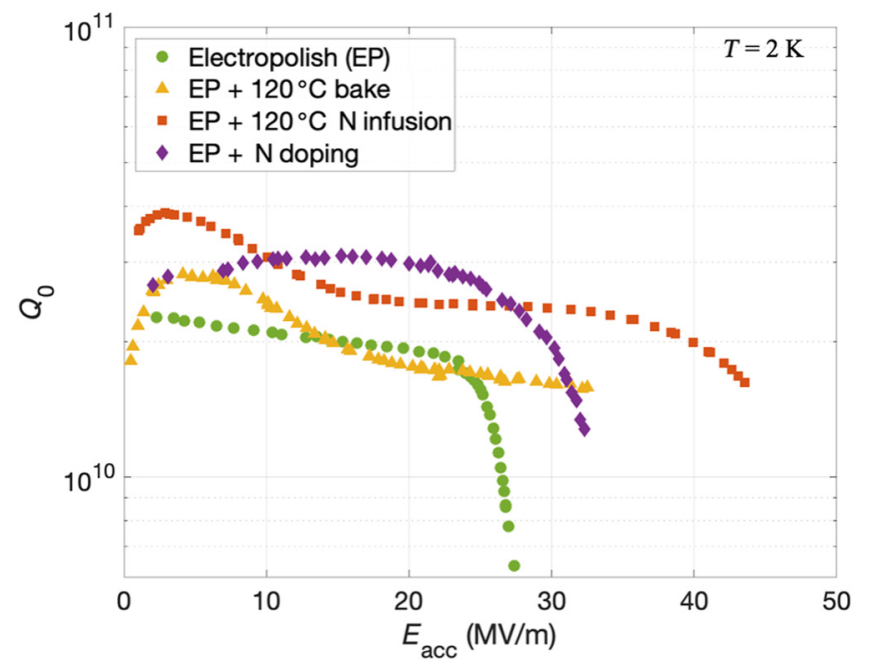

FIG. 1. Typical $Q_{0}$ vs $E_{\text {acc }}$ curves measured at $2.0 \mathrm{~K}$ for 1.3 GHz cavities treated with several standard surface treatments. Nitrogen infusion was developed relatively recently, and its ability to simultaneously produce high $Q_{0}$ and high gradients motivate an effort to improve the reproducibility of this treatment.

Previous studies have been performed on SRF cavities after heat treatment to dissolve the oxide. Palmer et al. performed studies on high-frequency $(\sim 9 \mathrm{GHz})$ cavities that had been vacuum heat treated both at temperatures in the $250-350{ }^{\circ} \mathrm{C}$ range as well as in the $1200-1400^{\circ} \mathrm{C}$ range to understand the effect of the oxide on SRF performance [13-16]. Eremeev performed studies on $1.5 \mathrm{GHz}$ cavities vacuum heat treated at temperatures of approximately $400{ }^{\circ} \mathrm{C}$ with a focus on the influence of the oxide on the high-field $Q$ slope (HFQS) [17]. In Sec. IV, results from these studies are compared with the new results, showing some interesting consistencies. However, it is also highlighted that the studies presented in this paper have significantly different conclusions about the causes for changes in performance and for practical potential of the oxide dissolution treatment.

In this paper, we frequently refer to the surface resistance $R_{s}$ when discussing how treatment modifies $Q_{0}$. Here $R_{s}$ is related (assuming $R_{s}$ is reasonably uniform over the surface) to $Q_{0}$ via a geometric factor $G$ through $R_{s}=G / Q_{0}$. At temperatures near the critical temperature $T_{c}, R_{S}$ is generally determined by the intrinsic properties of the superconductor ( $T_{c}$, coherence length $\xi$, etc.) in a strongly temperature-dependent component of the surface resistance based on the Bardeen-Cooper-Schrieffer (BCS) theory of superconductivity (referred to as the "BCS resistance" $R_{\mathrm{BCS}}$ ) [18-20]. The BCS resistance approaches zero for temperatures $T<<T_{c}$, and sources of surface resistance that are not intrinsic to the superconductor can begin to dominate, usually referred to as the residual resistance $R_{\text {res }}\left(R_{S}=R_{\mathrm{BCS}}+R_{\text {res }}\right)$. The residual resistance is known to be increased by extrinsic factors such as trapped flux and electron loading (e.g., field emission, multipacting), but an "intrinsic" component of the residual resistance is often discussed as well, with little understanding of its origin or expected value [18]. We show that the treatment methods described in this paper will modify $R_{\mathrm{BCS}}$ and $R_{\text {res }}$ in different ways, and may help to set an upper bound on the contribution of the "intrinsic" $R_{\text {res }}$.

\section{EXPERIMENTAL PROCEDURE}

For this study, several $1.3 \mathrm{GHz}$ single cell cavities made from bulk niobium are prepared with established SRF treatment methods (EP, $75 / 120^{\circ} \mathrm{C}$ bake, nitrogen doping) as a baseline. As is typical, the cavities are ultrasonically cleaned, high-pressure water rinsed (HPR), and assembled to vacuum hardware, including rf feedthroughs, a burst disc, and a pumpout port closed by a right angle valve. The assembled cavities are placed in a vertical test dewar and rf tested to establish baseline $Q_{0}$ vs $E_{\text {acc }}$ performance.

In the baseline test prior to heat treatment, the inner and outer surfaces of the cavities are expected to be covered by the natural oxide, which is composed primarily of $\mathrm{Nb}_{2} \mathrm{O}_{5}$. Surface studies of niobium samples have shown (e.g., via $\mathrm{x}$-ray photoelectron spectroscopy [14,17] or x-ray reflectivity [21]) that this oxide can be dissociated by vacuum heat treatment at temperatures above $300^{\circ} \mathrm{C}$ into $\mathrm{Nb}, \mathrm{O}$, and a small amount of residual suboxides.

After heat treatment, a new oxide layer will grow if the surface is then exposed to air or water, so cavities are heat treated while still assembled to vacuum hardware. After baseline measurement, an assembled cavity is transported to an oven, which uses forced convection of heated air to maintain an internal temperature of up to $300{ }^{\circ} \mathrm{C}$. All of the vacuum hardware is compatible with this temperature range except for the AlMg gaskets used to seal the cavities, so water cooling is used to lower the temperature of the flanges to an acceptable temperature. Heating bands around the beampipes of the cavity offset the water cooling so that the rf volume of the cavity is maintained in the above $300^{\circ} \mathrm{C}$ range, as monitored by thermocouples. To prevent absorption of atmospheric gases (including oxygen and hydrogen) in its exterior surface, cavities are installed in the oven inside a steel can, into which argon gas is purged at a constant rate. The argon atmosphere is sampled into an oxygen monitor, which typically reads less than 1000 ppm. The apparatus is shown in Fig. 2.

To carry out the heat treatment, the cavity temperature is ramped up from room temperature at a rate of approximately $2{ }^{\circ} \mathrm{C} / \mathrm{min}$ to the maximum temperature for approximately $2.5 \mathrm{~h}$ before turning off the heating power. Only a limited number of thermocouples are placed around the cavity (see Fig. 3), but the temperature range over the surface is expected to be in the range $250-400{ }^{\circ} \mathrm{C}$. Because this temperature range is between that used in the approximately $120^{\circ} \mathrm{C}$ low-temperature bake used to prevent 


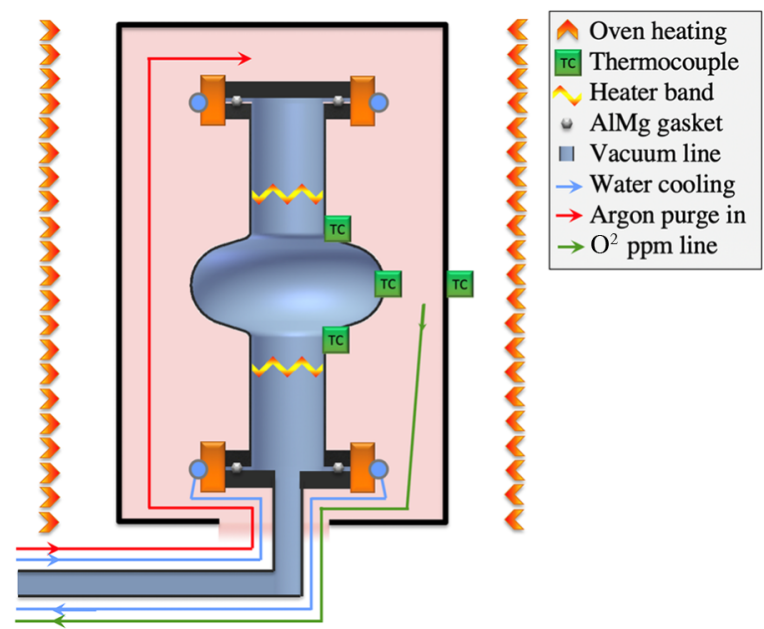

FIG. 2. Apparatus described in the text used to heat treat the niobium surrounding the elliptically shaped rf volume at a temperature higher than $300^{\circ} \mathrm{C}$. Precautions are taken to keep the $\mathrm{AlMg}$ gaskets cool and to prevent uptake of atmospheric gases in the exterior of the cavity wall.

HFQS [7,8] and the above $600{ }^{\circ} \mathrm{C}$ high-temperature furnace treatment used to degas hydrogen, we refer to this treatment as a "medium-temperature bake" (mid-T bake). During the mid-T bake, the cavity internal volume is pumped with a turbomolecular pump. For most of the cavities studied so far, after the mid-T bake, the oven is cooled down to room temperature directly. In the case of one cavity, TE1AES011, the mid-T bake is maintained at temperature for $22 \mathrm{~h}$, then the oven temperature is lowered to a hold at $120^{\circ} \mathrm{C}$, and the argon purge is stopped. The cavity

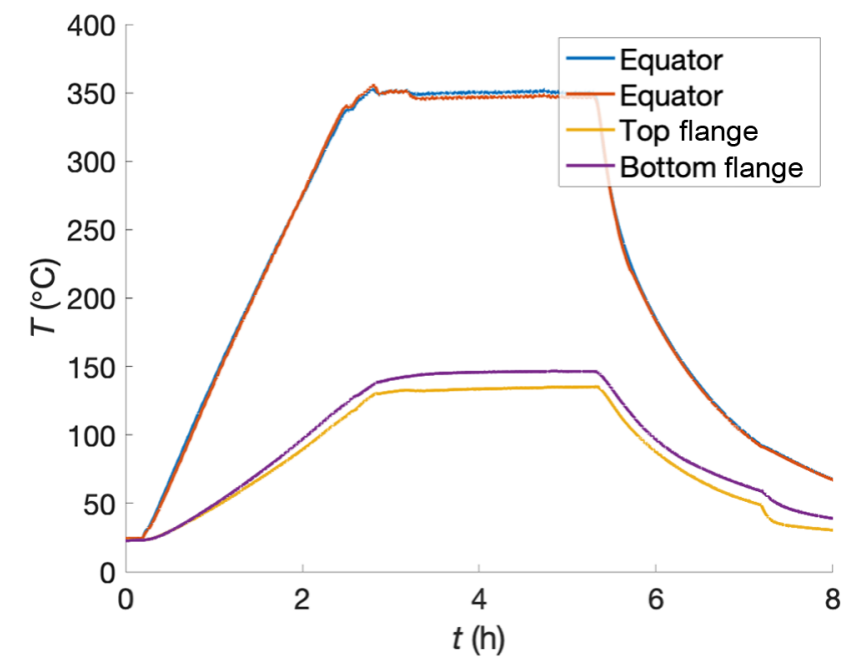

FIG. 3. Typical thermocouple readings during mid-T bake. Two sensors are located on the equator and one on each of the two cavity flanges, which are surrounded by water-cooled clamps. These locations are monitored, but it is expected that the temperature varies significantly between the equator, the heater-wrapped beamtubes, and the water-cooled flanges.

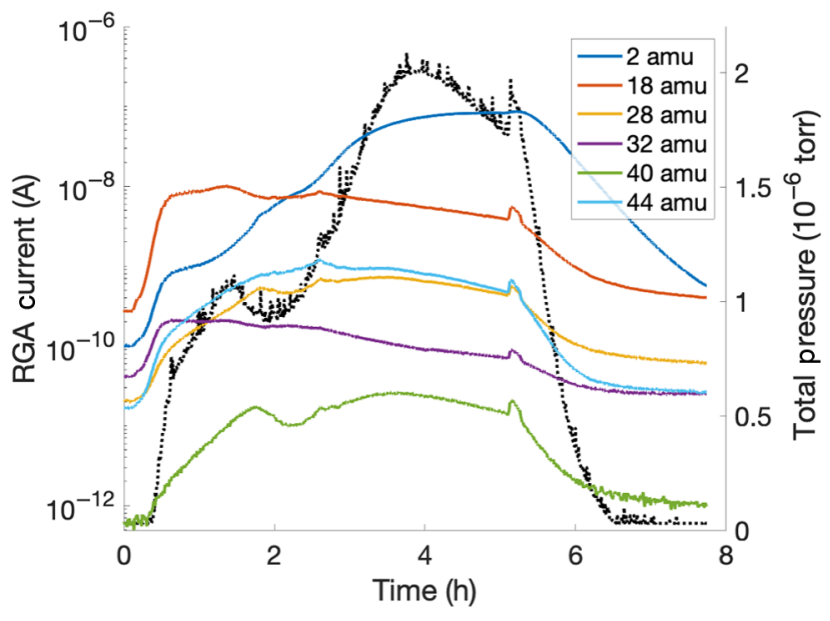

FIG. 4. Typical RGA readings during mid-T bake. The dotted line shows total pressure measured by the RGA and the solid lines at 2, 18, 28, 32, 40, and 44 amu are interpreted as indicators of hydrogen, water, nitrogen, oxygen, argon, and carbon dioxide. The peak observed at $\sim 5 \mathrm{~h}$ is likely caused by a brief temperature spike from the oven that occurs at the end of its program as it redirects airflow.

is backfilled with 25 mtorr of nitrogen via a needle valve (with the turbopump off) and this is maintained for $48 \mathrm{~h}$. This is done to mimic a $120^{\circ} \mathrm{C}$ nitrogen infusion.

A residual gas analyzer (RGA) on the pump cart is used to measure gas species in the cavity vacuum during the heat treatment (see Fig. 4) and to check the cavities for leaks before and after the heat treatment via spraying helium on vacuum connections. A leak was discovered in the vacuum line connected to the burst disc on TE1AES012 after it received heat treatment, suggesting that a leak opened during the treatment. It was a relatively small leak of approximately $1 \times 10^{-9}$ torr $1 / \mathrm{s}$, and tightening the bolts on the flange fixed the leak.

After mid-T bake, cavities are transported to the vertical test dewar for a second cryogenic rf test. None of the cavities in this study are disassembled or vented between the baseline $\mathrm{rf}$ test and this second rf test. However, after the second RF test, TE1PAV008 and TE1AES012 are vented with nitrogen gas after vertical test, partially disassembled, then given a high-pressure water rinse before reassembly and a third RF test. This is done to study the effect of reoxidizing the surface. TE1PAV005 is oxidized in a different way: it is vented with nitrogen, partially disassembled, left for 10 min exposed to cleanroom air, then reassembled and pumped out. TE1PAV008 is also treated with subsequent hydrofluoric acid (HF) rinses and remeasured.

\section{RADIO-FREQUENCY RESULTS: EP BASELINE}

The $Q_{0}$ vs $E_{\text {acc }}$ curves are obtained using the standard method of calibrated measurements of forward, reflected, 

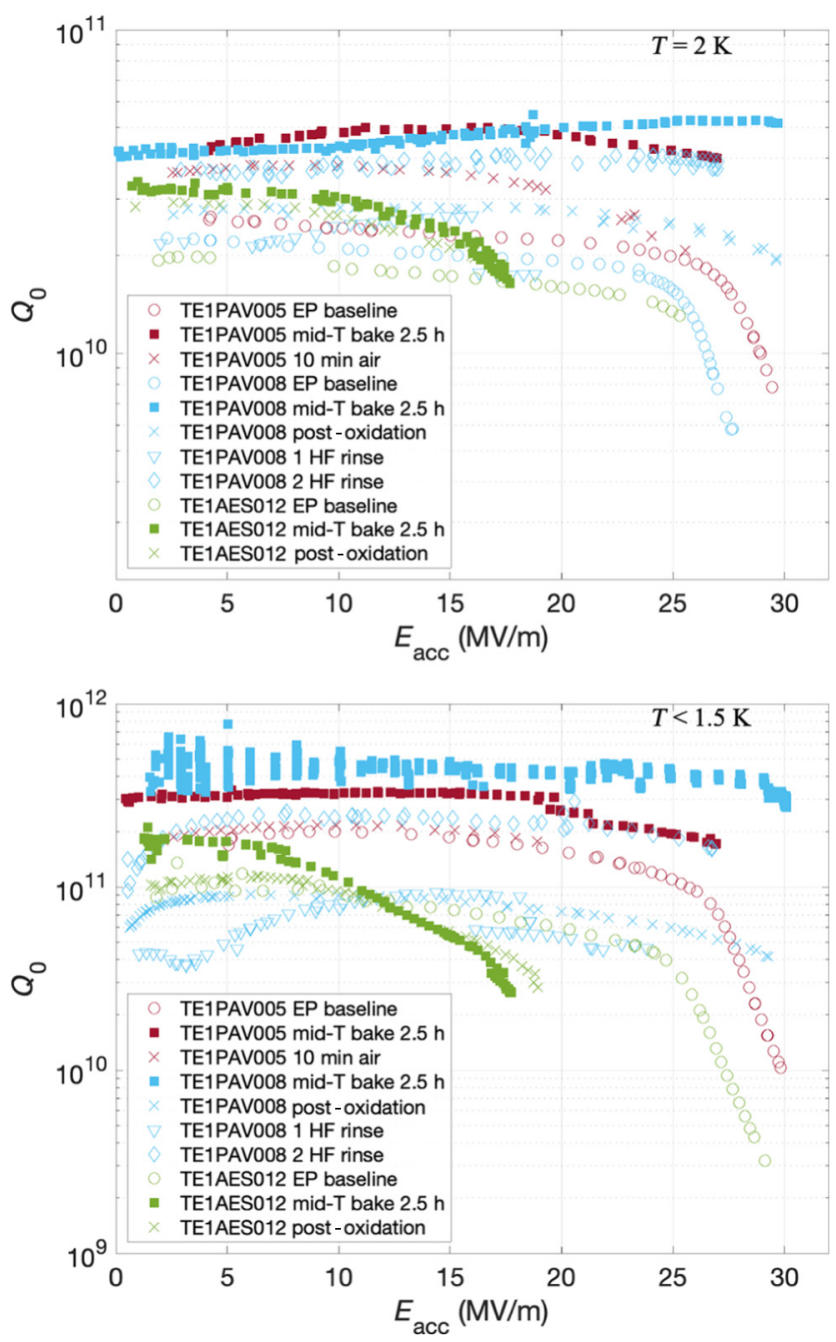

FIG. 5. Quality factor versus accelerating gradient in vertical cryogenic tests of the three EP baseline cavities treated with mid$\mathrm{T}$ bake. Measurements are performed at three stages: baseline, after mid-T bake, and after oxidation, except that no $T<1.5 \mathrm{~K}$ measurement is made on TE1PAV008 during baseline testing. Measurements are performed at a cavity temperature of $2.0 \mathrm{~K}$ (top) and 1.4-1.5 K (bottom).

and transmitted rf power into the cavity (for a description of the technique, see Ref. [18]). Measurements are made at $2.0 \mathrm{~K}$ - a typical operating temperature for a niobium $1.3 \mathrm{GHz}$ SRF cavity in an accelerator - and at a temperature in the $1.4-1.5 \mathrm{~K}$ range - close to the minimum temperature possible in the vertical test dewar, in a range where the BCS resistance is expected to be small compared with the residual resistance. To minimize $R_{\text {res }}$ owing to trapped flux dissipation, the cavities under measurement have all been previously heat treated at high temperatures to improve flux expulsion and are all cooled in a compensated magnetic field with strong thermal gradients [22-25]. The curves are shown in Fig. 5 for the three cavities that had EP baseline before heat treatment.
The $Q$ vs $E$ curves for the EP baseline cavities are fairly typical, including the observation of the HFQS in the typical gradient range $25-30 \mathrm{MV} / \mathrm{m}$.

After mid-T bake, extremely high-quality factors are observed in TE1PAV008 and TE1PAV005. At temperatures of $1.4-1.5 \mathrm{~K}$ (bottom of Fig. 5), $Q_{0}$ values in the range of $3-4 \times 10^{11}$ are observed up to gradients as high as $30 \mathrm{MV} / \mathrm{m}$. Although $Q_{0}$ values in the range of $2 \times 10^{11}$ have been reported previously [23,26,27], $Q_{0}$ above $3 \times 10^{11}$ at a gradient of approximately $20 \mathrm{MV} / \mathrm{m}$ is unprecedented. At $2.0 \mathrm{~K}$, the mid-T baked cavities show an unexpected anti- $Q$-slope behavior - an increase in $Q_{0}$ to around 3-4 $\times 10^{10}$ with increasing $E_{\text {acc }}$ in the range $5-15 \mathrm{MV} / \mathrm{m}$. This behavior, which is typically associated with nitrogen-doped cavities [9], is also unprecedented in this frequency and gradient range without nitrogen doping/infusion.

After TE1PAV008 is oxidized by exposure to air and water, its $Q_{0}$ degrades at all fields compared with immediately after mid-T bake, but is still higher than the EP baseline at $2 \mathrm{~K}$. The degradation is even smaller for TE1PAV005, which only received a $10 \mathrm{~min}$ exposure to air.

Both for the mid-T bake and the oxidized case, TE1AES012 showed $Q$-slope degradation not observed in the other two cavities. This may be related to the leak that is observed after the mid-T bake.

The gradient is limited in the baseline tests by HFQS. After mid-T bake, no HFQS is observed in either TE1PAV008 or TE1PAV005, though both cavities are limited by quench of approximately $30 \mathrm{MV} / \mathrm{m}$. TE1PAV005 also shows some $Q$-switch behavior in the gradient range 20-30 MV/m. Both cavities that had their oxides regrown after mid-T bake maintain very similar quench fields.

No x-rays above background are observed in any of the measurements except during some minor processing activity in TE1PAV005 in the test after $10 \mathrm{~min}$ air exposure.

No error bars are included in Fig. 5 for visual clarity, but we briefly discuss measurement uncertainty here. TE1PAV005 is tested with an input coupler with $Q_{\text {ext }}$ of $9 \times 10^{9}$ (each cavity is also tested with a pickup probe antenna, which is much more weakly coupled than the input and does not contribute strongly to uncertainty). This means that for the post-mid-T bake and post-oxidation $\mathrm{rf}$ measurements at less than $1.5 \mathrm{~K}$, the cavity is strongly overcoupled, increasing uncertainty. Under nominal conditions, the uncertainty in $Q_{0}$ and $E_{\text {acc }}$ is expected to be approximately $10 \%$, but in this case, with $Q_{0}$ significantly higher than $Q_{\text {ext }}$, the uncertainty is closer to $50 \%$. After seeing these results, much shorter antennas are used for the tests of TE1AES012 and TE1PAV005, with $Q_{\text {ext }} \sim 5 \times 10^{10}$. This is expected to reduce the uncertainty much closer to the $10 \%$ level. These estimates of uncertainty are based on several detailed studies in the literature, including Refs. [28-30]. 

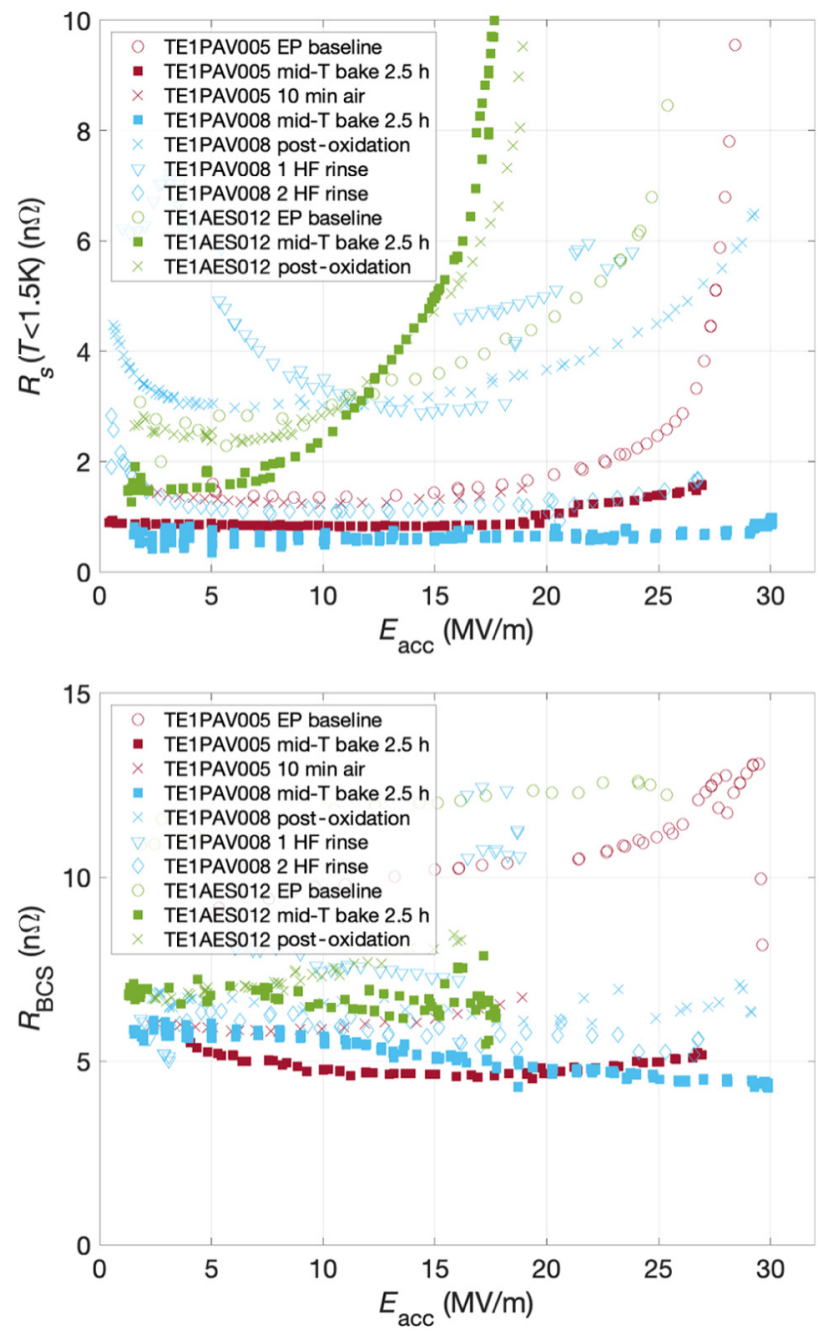

FIG. 6. Decomposed surface resistance of the three cavities from Fig. 5. Here $R_{\mathrm{BCS}}$ (bottom) is calculated by subtracting the surface resistance at $1.4-1.5 \mathrm{~K}$ (top) from the surface resistance at $2.0 \mathrm{~K}$.

The $Q$ vs $E$ curves are decomposed in Fig. 6. The surface resistance in the $1.4-1.5 \mathrm{~K}$ range is plotted in the top of the figure and $R_{\mathrm{BCS}}$ is plotted in the bottom, calculated by subtracting the $1.4-1.5 \mathrm{~K}$ resistance from the $2.0 \mathrm{~K}$ resistance.

A decrease in $R_{\mathrm{BCS}}$ as a function of field is observed in the gradient range $5-15 \mathrm{MV} / \mathrm{m}$. No decrease is observed in the less than $1.5 \mathrm{~K}$ curve. This suggests that the anti- $Q$ slope at $2.0 \mathrm{~K}$ originates from the BCS resistance, similar to the anti- $Q$ slope observed in doped cavities.

The plot of $R_{S}(T<1.5 \mathrm{~K})$ illustrates the extremely small surface resistance measured in TE1PAV008 and TE1PAV005. For TE1PAV005, surface resistance is measured as a function of temperature to extract the residual resistance by fitting $R_{\mathrm{BCS}}$ to Mattis-Bardeen theory $[19,31,32]$. The measurement is performed at $16 \mathrm{MV} / \mathrm{m}$ to show that the small residual resistance is achieved even at

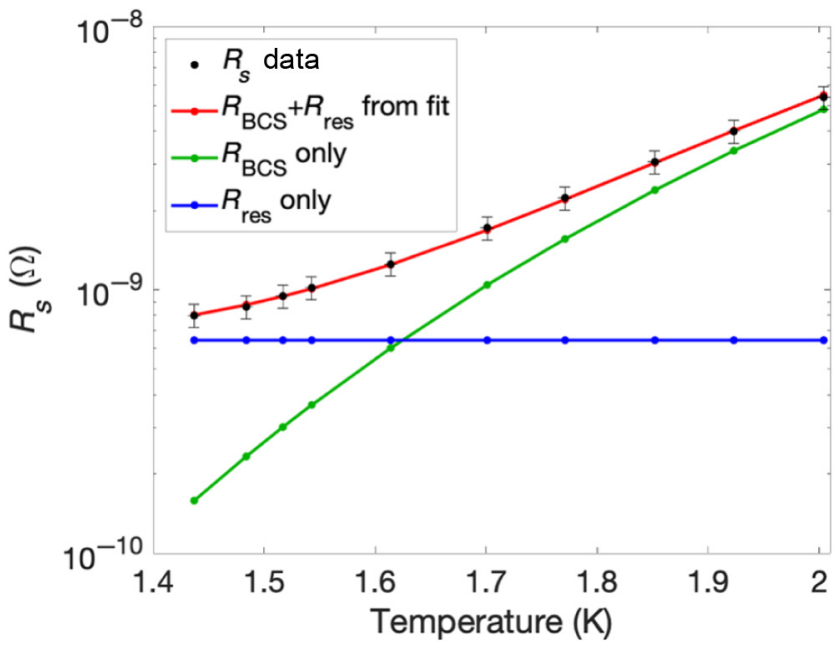

FIG. 7. Measurement of and fit to surface resistance versus temperature data at $16 \mathrm{MV} / \mathrm{m}$ for TE1PAV005 after mid-T bake. The fit gives a residual resistance of just $0.63 \pm 0.06 \mathrm{n} \Omega$.

useful accelerating fields. The result is shown in Fig. 7. The extrapolation to $R_{\mathrm{BCS}} \rightarrow 0$ from Mattis-Bardeen theory results in a residual resistance of just $0.63 \pm 0.06 \mathrm{n} \Omega$.

In addition to measurements of $R_{s}$ vs $T$, frequency $f$ vs $T$ is measured close to $T_{c}$, and the combined data are used to extract material properties of the superconductor [33]. The extracted values are in Table I, including data from TE1RI006, which is presented in Sec. IV. The critical temperature and energy gap values agree within uncertainty for all the treatments. The mean free path value for TE1PAV008 is somewhat higher than the others, but consistent with the variation observed in mean free path measurements observed in Ref. [34] for cavities treated with 2/6 nitrogen doping, which also produces mean free path values in this range. This suggests that the superconducting properties resulting from the mid-T bake are fairly consistent.

Sensitivity is measured for TE1PAV005 after mid-T bake by measuring $Q$ vs $E$ after cooling slowly in a $20 \mathrm{mG}$ field. The result is shown in Fig. 8. The sensitivity is higher than that of an electropolished cavity, resembling that of a nitrogen-doped cavity [25].

TABLE I. Critical temperature $T_{c}$, mean free path $l$, and reduced energy gap $\Delta / k_{B} T_{c}$ extracted from fits to measurements of $R_{s}$ and $f$ vs $T$ for cavities TE1PAV005, TE1PAV008, and TE1RI006 after mid-T bake.

\begin{tabular}{lccc}
\hline \hline Cavity & $T_{c}(\mathrm{~K})$ & $l(\mathrm{~nm})$ & $\Delta / k_{B} T_{c}$ \\
\hline TE1PAV005 & $8.94 \pm 0.15$ & $48 \pm 24$ & $2.07 \pm 0.03$ \\
TE1PAV008 & $9.04 \pm 0.15$ & $167 \pm 84$ & $2.10 \pm 0.03$ \\
TE1RI006 & $8.93 \pm 0.15$ & $62 \pm 31$ & $2.10 \pm 0.03$ \\
\hline \hline
\end{tabular}




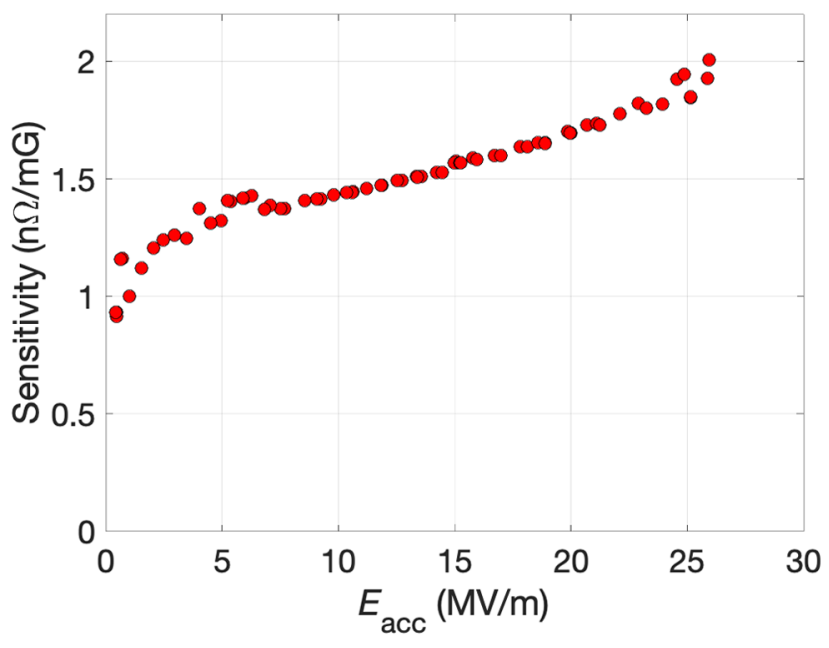

FIG. 8. Sensitivity to trapped flux as a function of accelerating field for TE1PAV005 after mid-T bake, prior to venting.

\section{RADIO-FREQUENCY RESULTS: $120^{\circ} \mathrm{C}$ AND NITROGEN-DOPED BASELINE}

The initial results of the mid-T bake encouraged additional studies. In addition to the three cavities that received EP baseline, three cavities are given mid-T bake that had a different baseline treatment. TE1RI006 is 3/60 nitrogen doped (furnace treated at $800^{\circ} \mathrm{C}$ with 3 min of nitrogen injection followed by $60 \mathrm{~min}$ of annealing) with $10 \mu \mathrm{m} \mathrm{EP}$. TE1PAV011 is given EP followed by a low-temperature bake at $120^{\circ} \mathrm{C}$ for $48 \mathrm{~h}$. TE1AES011 is given EP plus lowtemperature bake at $75^{\circ} \mathrm{C}$ for $4 \mathrm{~h}$ followed by $120^{\circ} \mathrm{C}$ for $48 \mathrm{~h}$. The mid-T bake of TE1AES011 is slightly different. It is given an extra-long 22-h mid-T bake, then the temperature is lowered to $120^{\circ} \mathrm{C}$, where the cavity is vented to $25 \mathrm{mtorr}$ of nitrogen, and it remained in this state for $48 \mathrm{~h}$. The $Q_{0}$ vs $E_{\text {acc }}$ curves of these cavities are shown in Fig. 9.

Similar to the EP baseline cavities, the $Q_{0}$ vs $E_{\text {acc }}$ data are decomposed into residual and BCS resistance in Fig. 10.

\section{MICROSCOPIC MEASUREMENTS}

Microscopy of mid-T-baked samples is challenging because the transfer of samples from a bake setup to a microscope must be done under vacuum to study the effect of the bake on the oxide. Therefore, to perform this study, electropolished niobium samples are put into a SIMS tool with an in situ heating stage that could reach the appropriate temperature range. Measurements are made both on EP baseline samples, on samples after mid-T bake, and samples after exposure to air. The results are shown in Fig. 11. The counts are given a point-by-point normalization to the signal collected for niobium.

Figure 11 plots the measured intensity of the niobium oxide ion $\mathrm{Nb}_{2} \mathrm{O}_{5}^{-}$. The $\mathrm{Nb}_{2} \mathrm{O}_{5}$ oxide layer is expected to
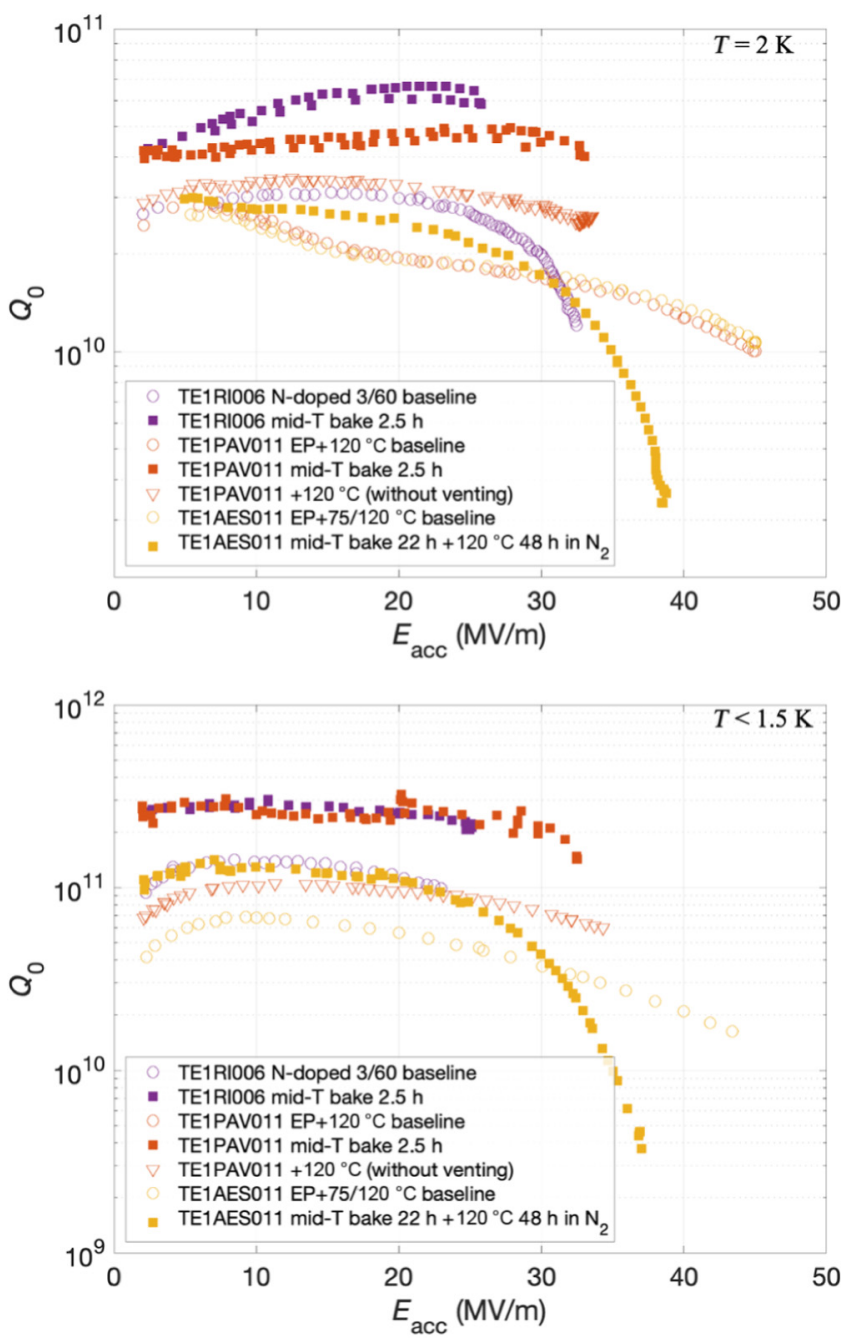

FIG. 9. Quality factor versus accelerating gradient in vertical test of three cavities treated with mid-T bake after baseline treatments of nitrogen doping or low-temperature baking. TE1AES011 is given additional low-temperature nitrogen treatment at $120^{\circ} \mathrm{C}$. The gradient is limited in each test by quench.

be $3-5 \mathrm{~nm}$ thick, and it appears to sputter in $30-50 \mathrm{~s}$. This gives an approximate calibration of sputter time to a depth of $10 \mathrm{~s} / \mathrm{nm}$. After the mid-T bake, the $\mathrm{Nb}_{2} \mathrm{O}_{5}^{-}$signal in the SIMS drops very quickly to the noise level, indicating a substantial reduction of the oxide layer. After exposing to air, the oxide regrows, though it appears to be somewhat thinner than the initially electropolished sample. This may be due to growing the oxide in air rather than in water.

Figure 12 shows the intensity of $\mathrm{NbN}^{-}$as a function of depth as a measurement of nitrogen interstitials in the niobium bulk. An unexpected increase in the intensity of the $\mathrm{NbN}^{-}$signal is observed after the mid-T bake. A bump, some tens of nanometers deep, appears close to the surface, similar to a nitrogen-infused sample. It persists after the oxide is regrown. The figure also compares this bump 

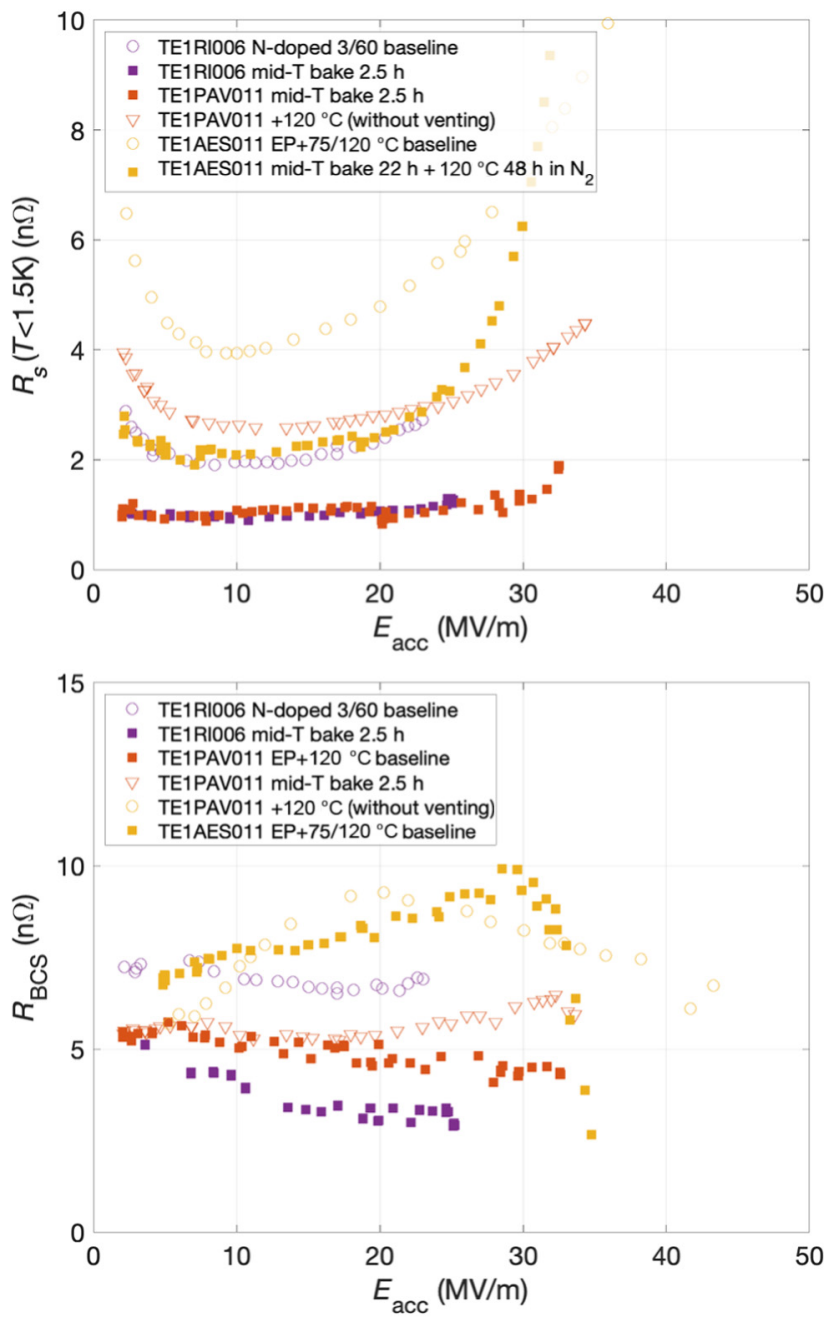

FIG. 10. Decomposed surface resistance of the three cavities from Fig. 9. Here $R_{\mathrm{BCS}}$ (bottom) is calculated by subtracting the surface resistance at $1.4-1.5 \mathrm{~K}$ (top) from the surface resistance at $2.0 \mathrm{~K}$.

with that observed in a sample that is separately treated in a vacuum furnace with a $120^{\circ} \mathrm{C}$ infusion.

\section{DISCUSSION}

\section{A. Effect of surface condition on $\mathrm{rf}$ performance}

One of the changes consistently observed after mid-T bake is a decrease in residual resistance. Below $1.5 \mathrm{~K}$, $R_{s}$ values are regularly observed in the range of $1 \mathrm{n} \Omega$ or less. This may be related to the dissolution of the $\mathrm{Nb}_{2} \mathrm{O}_{5}$ layer, confirmed by SIMS measurements. A contribution by the natural oxide to residual resistance of some tenths of a nanoohm would be consistent with the measurements presented here. An increase in residual resistance with oxide thickness is also consistent with measurements on anodized cavities in the relevant gradient range [35]. The reduced residual resistance may have also been due to the diffusion of impurities. For example, nitrogen doping and

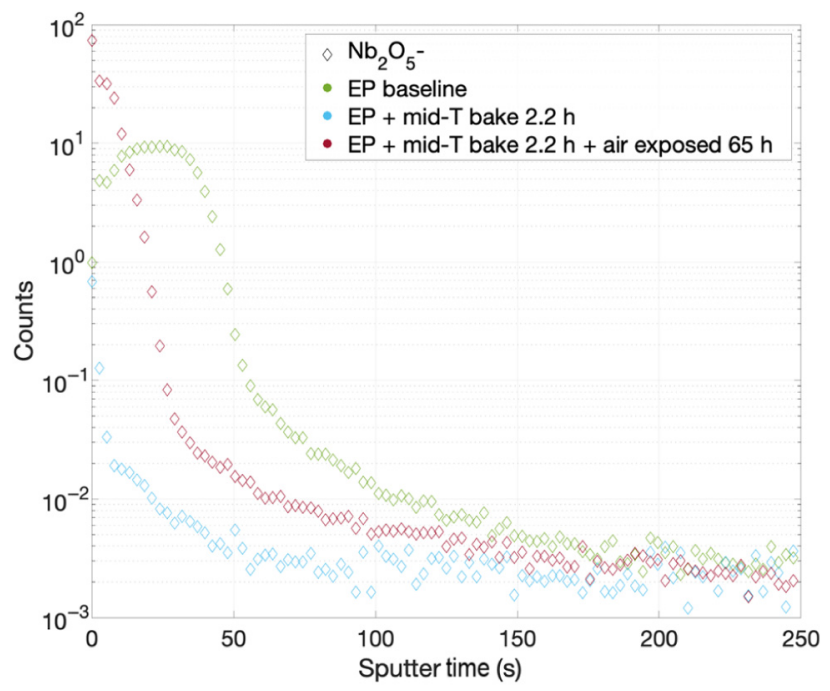

FIG. 11. SIMS depth profile measurements of $\mathrm{Nb}_{2} \mathrm{O}_{5}^{-}$in a niobium sample that is first given EP baseline, then given mid-T bake, then exposed to air. The $y$ axis is normalized point by point to the niobium signal.

nitrogen infusion are also known to cause a decrease in the residual resistance in this gradient range $[9,10]$. There is also a theory that suggests a contribution to residual resistance from direct phonon excitation (see, e.g., Ref. [36]), which may be reduced by oxide dissolution.

The very low value of residual resistance measured contributes to a limited body of knowledge around understanding of the "intrinsic" residual resistance (i.e., not caused by extrinsic factors such as trapped flux). By minimizing the effects of trapped flux (by minimizing the ambient field

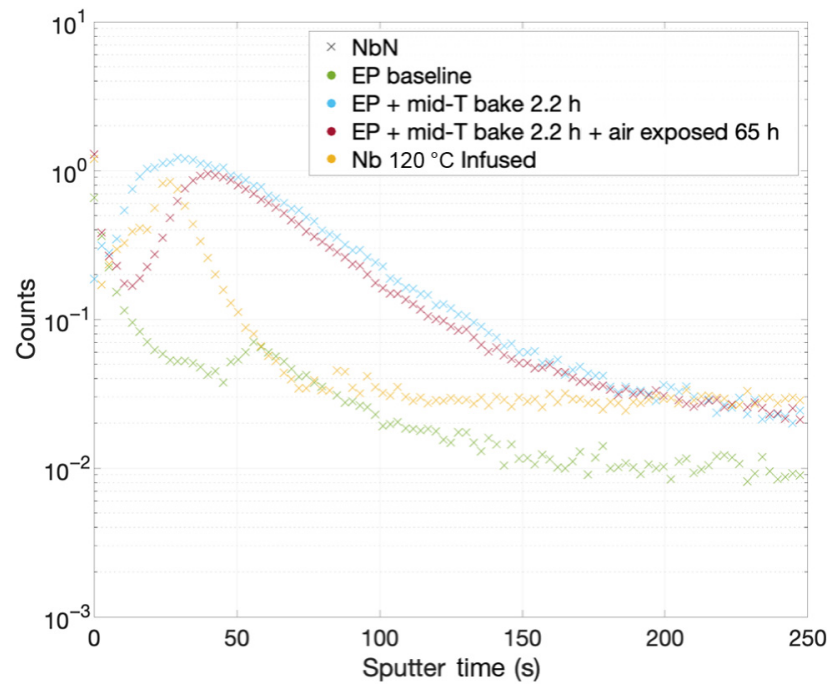

FIG. 12. Comparison of nitrogen signal in SIMS data between EP baseline, mid-T bake (with and without air exposure), and $120^{\circ} \mathrm{C}$ infused sample. The $y$ axis is normalized point by point to the niobium signal. 
during cooldown and applying flux expulsion) and minimizing the effect of the oxide (by removing or significantly reducing it), we place an upper bound on the contribution of the "intrinsic" residual resistance of just $0.6 \mathrm{n} \Omega$ for a cavity of this type.

The rf results consistently showed a decrease in BCS resistance after mid-T bake, and frequently showed anti$Q$ slope. This unexpected result may be due to nitrogen interstitials, as suggested by the bump observed in the $\mathrm{NbN}$ signal observed by SIMS after the mid-T bake. The bump is consistent with a nitrogen infusion, though no nitrogen gas is introduced during the heat treatment in the SIMS. It is possible that the nitrogen diffused into the niobium from elsewhere, such as from residual gases in the vacuum volume (including those degassed from surfaces other than that of the cavity), from surface contamination, from the bulk. Other possible sources of BCS modification include $\mathrm{O}$ and $\mathrm{C}$ interstitials modifying the mean free path. The higher sensitivity and lower mean free path are consistent with measurements of nitrogen-doped/infused cavities $[9,10]$.

The quench field for most of the cavities after mid-T bake is in the range $25-30 \mathrm{MV} / \mathrm{m}$. The consistency of this quench field is reminiscent of quenches in nitrogen-doped cavities, which have been observed to cluster in a narrow gradient range [37]. The only cavity with a quench field higher than this is TE1AES011, which, after the mid-T bake, is the only cavity that is backfilled to 25 mtorr with nitrogen and then received a $120^{\circ} \mathrm{C}$ heat treatment. This cavity reached $39 \mathrm{MV} / \mathrm{m}$ before quenching. This shows progress towards the goal of developing a treatment that achieves high gradients, but is more robust than nitrogen infusion. Additional exploration of the parameter space (time, temperature, nitrogen pressure) may help to increase the field further. The slope that is observed is shallower than typical HFQS - additional studies of the parameters space may help to develop understanding in the cause of the slope and prevent it in future treatments.

TE1AES012 shows substantially poorer performance after mid-T bake compared with the other cavities. This may be linked to the leak during the heat treatment, allowing atmospheric gases into the cavity even with the argon purge. For example, this could have allowed undesirable oxides to form.

TE1PAV008 shows the effect of reoxidation of the surface by exposure to water. BCS resistance stays roughly the same, though possibly increased slightly, consistent with the continued presence of nitrogen impurities in the rf layer. Residual resistance increases significantly, but it appears that this can be ameliorated by two HF rinses to return to approximately $2 \mathrm{n} \Omega$, similar to the post-EP surface of TE1PAV005 or the nitrogen-doped surface of TE1RI006. This is consistent with the residual resistance increase observed in a $120^{\circ} \mathrm{C}$ baked cavity followed by subsequent HF rinsing [38].

\section{B. Previous studies}

Palmer et al. previously performed studies on vacuum heat treatment to study the effect of the oxide. Palmer et al.'s experiments included firing cavities in a vacuum furnace at temperatures in the $1200-1400^{\circ} \mathrm{C}$ range, rf testing them without exposing to air, oxidizing the surfaces, heat treating in vacuum at temperatures $\sim 300^{\circ} \mathrm{C}$ for 5-10 min, and then rf testing again without exposing to air. The cavities Palmer worked with were substantially higher frequency, $8.6 \mathrm{GHz}$, which makes comparison of results complicated, but still interesting. Palmer et al.'s results show the lowest residual resistance after $1200-1400{ }^{\circ} \mathrm{C}$ firing and before exposing to oxygen. Here $R_{\text {res }}$ was found to have a similar small value after oxidation and heat treatment to $350{ }^{\circ} \mathrm{C}$ for $10 \mathrm{~min}$, but heat treatment at $300^{\circ} \mathrm{C}$ or $325^{\circ} \mathrm{C}$ resulted in significant increases in the residual resistance. This suggests a strong temperature dependence, and may also imply some dependence on time at these temperatures. The residual resistance value after $350^{\circ} \mathrm{C}$ heat treatment was smaller than the value after initial bulk chemistry, in agreement with the results presented here. Interestingly, Palmer et al.'s results also showed a decrease in BCS resistance after $325-350^{\circ} \mathrm{C}$ heat treatment, also consistent with the results presented here. They propose that diffusion of oxygen causes a change in mean free path that, in turn, brings the BCS resistance closer to a minimum. The role of nitrogen was not discussed [13-16].

Eremeev also performed previous studies of the effect of heat treatment in vacuum at $\sim 400{ }^{\circ} \mathrm{C}$ to study the effect of the oxide on HFQS. Owing to the focus of these experiments, it is difficult to compare results: measurements were performed only at $1.5 \mathrm{~K}$, so BCS resistance at $2 \mathrm{~K}$ cannot be extracted; there are limited comparisons with data before heat treatment at around $400{ }^{\circ} \mathrm{C}$ in vacuum; and the residual resistance is relatively high even before heat treatment $\left(Q_{0} \sim 3 \times 10^{10}\right.$ at low fields at $\left.1.5 \mathrm{~K}\right)$ [17].

\section{Outlook: future improvement and applications}

Future work will focus on parameter space exploration with the goal of improving performance. Possible avenues to explore will include trying to optimize the nitrogen profile using feedback between SIMS studies, light removal post-mid-T bake, and cavity testing. Treatments with the best performance will be evaluated for reliability, ideally including studies at other labs. In addition, cavities at other frequencies and multicells will be treated with the mid-T bake. In addition to possible applications, studies of this treatment may help to improve understanding of "intrinsic" residual resistance and of the $Q_{0}$ improvement brought about by nitrogen doping and infusion.

For application in accelerators, it would be very challenging to use cavities in accelerators after mid-T bake, but before exposure to air. It seems more likely that oxidized 
surfaces would have to be used in accelerator applications. The performance of TE1PAV005 after two HF rinses shows that this is a promising possibility. With $Q_{0}$ of $3.9 \times 10^{10}$ at $27 \mathrm{MV} / \mathrm{m}$, this cavity would significantly exceed the planned specifications for LCLS-II HE, making it a possible treatment to study for future high-duty-factor applications.

In addition, there are nonaccelerator applications requiring extremely high $Q_{0}$ electromagnetic resonators where exposure to air after heat treatment is not needed or else where only a very brief exposure is needed (duration similar to that used for TE1PAV005). These include applications such as quantum computing [35,39] and quantum sensors.

\section{CONCLUSIONS}

In this study, several $1.3 \mathrm{GHz}$ single-cell cavities are first assembled in a cleanroom to $\mathrm{rf}$ hardware and put under vacuum, then given a mid-T bake: a heat treatment for several hours at a temperature of $250-400{ }^{\circ} \mathrm{C}$, which is expected to be sufficient to remove or significantly reduce the oxide. The mid-T bake is found to (1) significantly decrease residual resistance, and (2) significantly reduce $\mathrm{BCS}$ resistance at $2 \mathrm{~K}$ relative to an electropolished cavity. Here $Q_{0}$ values of $3-4 \times 10^{11}$ are measured at less than 1.5 and $20 \mathrm{MV} / \mathrm{m}$, higher than has been reported previously in the literature for such conditions, and a residual resistance of just $0.63 \pm 0.06 \mathrm{n} \Omega$ is measured at $16 \mathrm{MV} / \mathrm{m}$. After exposing the cavities to air and water, the $Q_{0}$ degraded somewhat but is still approximately $3 \times 10^{10}$ at $2 \mathrm{~K}$, similar to nitrogen-doped cavities. The observation of anti- $Q$ slope in the BCS resistance, the increased sensitivity to trapped flux, and the nitrogen observed in SIMS are all consistent with the $Q_{0}$ of the cavity being enhanced owing to the presence of nitrogen interstitials. HF rinse studies so far show some potential to improve $Q_{0}$ at $2 \mathrm{~K}$ to nearly $4 \times 10^{10}$ at $27 \mathrm{MV} / \mathrm{m}$. The quench field is found to be fairly consistently in the $25-30 \mathrm{MV} / \mathrm{m}$ range after $2.5 \mathrm{~h}$ of mid$\mathrm{T}$ bake, but the addition of a $120^{\circ} \mathrm{C}$ step for $48 \mathrm{~h}$ with 25 mtorr of nitrogen, similar to nitrogen infusion is found to increase the quench field to $39 \mathrm{MV} / \mathrm{m}$. Future studies will focus on continued optimization of the process as well as exploration of other cavity frequencies and multicells.

\section{ACKNOWLEDGMENTS}

The authors would like to thank B. Tennis for preparation of the mid-T-baking setup as well as the Fermilab SRF processing and VTS teams. Thanks go to Curtis Crawford, Hasan Padamsee, Martina Martinello, and Daniel Bafia for useful discussions. This work was supported by the United States Department of Energy, Offices of High Energy Physics and Basic Energy Sciences under Contract No. DE-AC05-06OR23177.
[1] R. Brinkmann, B. Faatz, K. Flöttmann, J. Rossbach, J. R. Schneider, H. Schulte-Schrepping, D. Trines, T. Tschentscher, and H. Weise, TESLA XFEL, First Stage of the X-Ray Laser Laboratory, Technical Design Report (Hamburg, 2001).

[2] J. N. Galayda (ed.), LCLS-II Final Design Report, LCLSII1.1-DR-0251-R0 (2015).

[3] M. Ball, A. Burov, B. Chase, A. Chakravarty, A. Chen, S. Dixon, J. Edelen, A. Grassellino, D. Johnson, S. Holmes, S. Kazakov, A. Klebaner, I. Kourbanis, A. Leveling, O. Melnychuk, D. Neuffer, T. Nicol, J. Ostiguy, et al., The PIP-II Conceptual Design Report (2017).

[4] O. S. Brüning, J. Poole, P. Collier, P. Lebrun, R. Ostojic, S. Myers, and P. Proudlock, LHC Design Report (Geneva, 2004).

[5] T. E. Mason, Spallation Neutron Source Project Completion Report (2006).

[6] S. Peggs (ed.), European Spallation Source: Conceptual Design Report (Lund, 2012).

[7] B. Visentin, J. Charrier, B. Coadou, and D. Roudier, Cavity baking: A cure for the high accelerator field Q0 drop, Proc. Ninth Work. RF Supercond. 198 (1999).

[8] P. Kneisel, Preliminary experience with 'in-situ' baking of niobium cavities, Proc. Ninth Work. RF Supercond. 328 (1999).

[9] A. Grassellino, A. Romanenko, D. Sergatskov, O. Melnychuk, Y. Trenikhina, A. Crawford, A. Rowe, M. Wong, T. Khabiboulline, and F. Barkov, Nitrogen and argon doping of niobium for superconducting radio frequency cavities: A pathway to highly efficient accelerating structures, Supercond. Sci. Technol. 26, 102001 (2013).

[10] A. Grassellino, A. Romanenko, Y. Trenikhina, M. Checchin, M. Martinello, O. S. Melnychuk, S. Chandrasekaran, D. A. Sergatskov, S. Posen, A. C. Crawford, S. Aderhold, and D. Bice, Unprecedented quality factors at accelerating gradients up to $45 \mathrm{MVm}-1$ in niobium superconducting resonators via low temperature nitrogen infusion, Supercond. Sci. Technol. 30, 094004 (2017).

[11] K. Umemori, T. Dohmae, M. Egi, Y. Hori, E. Kako, T. Konomi, S. Michizono, T. Saeki, H. Sakai, Y. Yamamoto, S. Kurosawa, K. Takeichi, J. Kamiya, and T. Okada, in Proc. 29th Linear Accel. Conf., Beijing, China, no. TUPO071, (2018).

[12] M. Wenskat, A. Prudnikava, D. Reschke, and J. Schaffran, in Proc. Eighteenth Conf. RF Supercond., Lanzhou, China, no. THPB007 (2017), pp. 759-761.

[13] F. L. Palmer, Influence of oxide layers on the microwave surface resistance of niobium, IEEE Trans. Magn. 23, 1617 (1987).

[14] F. L. Palmer, R. E. Kirby, F. K. King, and E. L. Garwin, Oxide overlayers and the superconducting properties of yttrium-processed Rf Nb, Nucl. Instruments Methods Phys. Res. A 297, 321 (1990).

[15] F. Palmer and M. Tigner, Effect of oxide layer on microwave surface resistance of superconducting niobium, IEEE Trans. Magn. 21, 1011 (1985).

[16] F. Palmer, in Proceedings of The Third Workshop on RF Superconductivity, Argonne National Laboratory, Illinois, USA, (1987), pp. 309-330.

[17] G. Eremeev, PhD Thesis, Cornell Univ., 2008. 
[18] H. Padamsee, J. Knobloch, and T. Hays, RF Superconductivity for Accelerators (Wiley-VCH, New York, 2008).

[19] D. C. Mattis and J. Bardeen, Theory of the anomalous skin effect in normal and superconducting metals, Phys. Rev. 111, 412 (1958).

[20] J. Bardeen, L. N. Cooper, and J. R. Schrieffer, Theory of superconductivity, Phys. Rev. 108, 1175 (1957).

[21] M. Delheusy, A. Stierle, N. Kasper, R. P. Kurta, A. Vlad, H. Dosch, C. Antoine, A. Resta, E. Lundgren, and J. Andersen, X-ray investigation of subsurface interstitial oxygen at $\mathrm{Nb}$ /oxide interfaces, Appl. Phys. Lett. 92, 1 (2008).

[22] A. Romanenko, A. Grassellino, O. Melnychuk, and D. A. Sergatskov, Dependence of the residual surface resistance of superconducting radio frequency cavities on the cooling dynamics around Tc, J. Appl. Phys. 115, 184903 (2014).

[23] A. Romanenko, A. Grassellino, A. C. Crawford, D. A. Sergatskov, and O. Melnychuk, Ultra-high quality factors in superconducting niobium cavities in ambient magnetic fields up to $190 \mathrm{mG}$, Appl. Phys. Lett. 105, 234103 (2014).

[24] S. Posen, M. Checchin, A. C. Crawford, A. Grassellino, M. Martinello, O. S. Melnychuk, A. Romanenko, D. A. Sergatskov, and Y. Trenikhina, Efficient expulsion of magnetic flux in superconducting radiofrequency cavities for high Q0 applications, J. Appl. Phys. 119, 213903 (2016).

[25] M. Martinello, A. Grassellino, M. Checchin, A. Romanenko, O. Melnychuk, D. A. Sergatskov, S. Posen, and J. F. Zasadzinski, Effect of interstitial impurities on the field dependent microwave surface resistance of niobium, Appl. Phys. Lett. 109, 062601 (2016).

[26] H. Safa, in Proc. 10th Work. RF Supercond. (2001), pp. 279.

[27] P. Kneisel, R. W. Roth, and H. G. Kiirschner, Results from a nearly 'defect-free' niobium cavity, Proc. Seventh Work. RF Supercond. 449 (1995).

[28] O. Melnychuk, A. Grassellino, and A. Romanenko, Error analysis for intrinsic quality factor measurement in superconducting radio frequency resonators, Rev. Sci. Instrum. 85, 124705 (2014).

[29] J. P. Holzbauer, Y. Pischalnikov, D. A. Sergatskov, W. Schappert, and S. Smith, Systematic uncertainties in
RF-based measurement of superconducting cavity quality factors, Nucl. Instruments Methods Phys. Res. Sect. A Accel. Spectrometers, Detect. Assoc. Equip. 830, 22 (2016).

[30] T. Powers, Theory and practice of cavity RF test systems, Proc. SRF 2005, 40 (2005).

[31] J. Halbritter, FORTRAN-program for the Computation of the Surface Impedance of Superconductors (Intern. Note KFZ Karlsruhe 3/70-6, 1970).

[32] J. Halbritter, Comparison between measured and calculatedRF losses in the superconducting state, Zeitschrift fur Phys. 238, 466 (1970).

[33] S. Meyers, S. Posen, and M. Liepe, in Proc. 27th Linear Accel. Conf., no. TUPP018 (2014).

[34] M. Martinello, A. Grassellino, M. Checchin, A. Romanenko, O. Melnychuk, D. A. Sergatskov, S. Posen, and J. F. Zasadzinski, Supplementary material for 'effect of interstitial impurities on the field dependent microwave surface resistance of niobium, Appl. Phys. Lett. 109, 1 (2016) of supplemental material (2016).

[35] A. Romanenko and D. I. Schuster, Understanding Quality Factor Degradation in Superconducting Niobium Cavities at Low Microwave Field Amplitudes, Phys. Rev. Lett. 119, 1 (2017)

[36] E. P. Kartheuser and S. Rodriguez, Residual surface resistance of superconductors at microwave frequencies, Appl. Phys. Lett. 24, 338 (1974).

[37] A. Grassellino, A. Romanenko, S. Posen, Y. Trenikhina, O. Melnychuk, D. A. Sergatskov, M. Merio, M. Checchin, and M. Martinello, in Proc. Seventeenth Int. Conf. RF Supercond., no. MOBA06 (2015).

[38] A. Romanenko, A. Grassellino, F. Barkov, and J. P. Ozelis, Effect of mild baking on superconducting niobium cavities investigated by sequential nanoremoval, Phys. Rev. Spec. Top. - Accel. Beams 16, 1 (2013).

[39] A. Romanenko, R. Pilipenko, S. Zorzetti, D. Frolov, M. Awida, S. Posen, and A. Grassellino. "Three-dimensional superconducting resonators at $\mathrm{T}<20 \mathrm{mK}$ with the photon lifetime up to tau=2 seconds," arXiv Prepr., no. 1810.03703, pp. 3-6 (2018). 